\title{
Utilization of obstetric analgesia in labor pain management and associated factors among obstetric caregivers in public health facilities of Kembata Tembaro Zone, Southern Ethiopia
}

This article was published in the following Dove Press journal: Journal of Pain Research

\section{Teketel Ermias Geltore' Ayanos Taye ${ }^{2}$ \\ Abraham Getachew Kelbore $^{3}$}

'Midwifery Department, School of Nursing and Midwifery, College of Health Sciences and Medicine, Wolaita Sodo University, Wolaita Sodo, Ethiopia; ${ }^{2}$ Nursing Department, School of Nursing and Midwifery, College of Health Sciences and Medicine, Jimma University, Jimma, Ethiopia; ${ }^{3}$ Dermatology Department, College of Health Sciences and Medicine, School of Medicine, Wolaita Sodo University, Wolaita Sodo, Ethiopia
Correspondence: Abraham Getachew Kelbore

Dermatology Department, College of Health Sciences and Medicine, School of Medicine, Wolaita Sodo University, P.O. Box 138, Wolaita Sodo, Ethiopia

Tel +25I 910328658

Email kelbore2005@gmail.com
Background: Nowadays, obstetric analgesia is provided routinely in most developed countries. However, in developing countries, labor pain management is not a well-established service. The poor utilization of labor analgesia in low-income countries including Ethiopia results in laboring mothers in unmeasured suffering, let alone analgesia. The aim of this study was to assess utilization of obstetric analgesia in labor pain management and associated factors among obstetric caregivers in public health facilities of KTZ, Kembata Tembaro zone, Southern Ethiopia.

Materials and methods: A facility-based cross-sectional study design was conducted among all obstetric caregivers at KTZ in public health facilities from March to April 2017. Data were collected by a pretested, self-administered, and structured questionnaire. A convenient sampling technique was used and descriptive analysis was done to characterize the study population. Bivariate and multivariate logistic regressions were used to identify factors associated with utilization of labor analgesia. The adjusted odds ratio with $95 \% \mathrm{CI}$ was used to show the strength of the association and a $P$-value $<0.05$ was used to declare the cutoff point in determining the level of significance.

Results: Three hundred forty respondents participated in the study with a response rate of $93 \%$. The prevalence of labor analgesia use by the respondents was $37.9 \%$. On multivariable logistic regression analyses, inadequate knowledge, positive attitude, and unavailability of labor analgesia drugs were significant independent predictors of obstetric analgesia utilization.

Conclusion: Proportion of analgesia utilization was low; inadequate knowledge, positive attitude, and unavailability of analgesia drugs in the facilities were significantly associated with obstetrics analgesia utilization. Health institutions and health personnel should work on provision of training for those obstetric care providers, and necessary drugs should be available in each facility.

Keywords: pain management, analgesia, obstetric caregiver, Ethiopia, institution based

\section{Background}

Labor is characterized by regular, painful uterine contractions that increase in frequency and intensity in three stages of labor. ${ }^{1}$ During labor, pain originates from different sites during each stage of the labor, which is a physiological phenomenon and its evolution is associated with ischemia of the uterus during contraction, effacement, dilation of 
cervix, stretching of the vagina, perineum, and compression of pelvic structures..$^{2-4}$

Women's experience of pain during labor greatly varies from feeling of little pain to extremely distressing pain. Hence, reliving labor pain is one of the main concerns for pregnant women, her families, and health care providers. So effective utilization of labor analgesia has implications on the course of labor, best outcomes, and the quality of obstetric care. $^{2,5}$ In high-income countries, many pharmacological and nonpharmacological treatments have been developed to alleviate labor pain. Among the methods, systemic opioids, nonopioids, epidural analgesia, combined spinal-epidural analgesia, inhalation agents, pudendal block, transcutaneous electrical nerve stimulation, massage, acupuncture, water immersion, yoga, music therapy, biofeedback, continuous support, positioning, ambulation, hypnosis, and breathing technique are used to manage labor pain.,

The effectiveness of these methods varies, but epidural analgesia remains the safest. It is the widely used analgesia that provides almost complete labor pain relief (90\%) with a favorable birth experience. ${ }^{8}$ In Ethiopia, the practice of labor pain management is very low. In 2014, the Ethiopian Food, Medicine, and Health Care Administration and Control Authority (FMHACA) has developed and implemented standard treatment guidelines. Giving analgesics and anesthetics to pregnant mothers without affecting maternal and fetal condition must be a concern to the health care provider. Provision of physical and psychological support and use of pharmacological methods are described to manage labor pain. ${ }^{9}$ The aim of pain relief in labor is to make an emotionally satisfying experience where a woman is delivering a healthy baby with as little distress, pain, and exhaustion as possible and with minimal risk to both mother and fetus. ${ }^{10}$

Labor pain is as old as human being, which is not simple but a complex phenomenon with sensory, emotional, and perceptive components and considered as one of the most serious kinds of pain. Although severe pain during labor is not life-threatening for a healthy parturient, it may result in the stimulation of the sympathetic nervous system leading to maternal hypertension and oxygen consumption affecting uteroplacental blood flow. Besides this, it can contribute to postpartum depression and posttraumatic stress disorder. However, most of these pain effects are alleviated by effective pain relief methods that may benefit the mother and fetus. ${ }^{6,11-13}$

For most women, labor causes severe pain, similar in degree to pain caused by the amputation of a finger. According to American College of Obstetricians and Gynecolo- gists, maternal request represents sufficient justification for pain relief and it is not considered acceptable for a client to experience untreated severe pain, while under a physician's care. ${ }^{14-16}$ In developed countries, analgesia is widely utilized for pain relief and they also focused on choice of methods and complication; however, in developing countries the issue concentrates on awareness, acceptability, and availability. ${ }^{13}$

On the other hand, the major factors that affect the utilization of obstetric analgesia in developing countries by caregivers are unavailability of drugs, health care delivery systems, knowledge, and religion. Of these, knowledge, attitudes, and skills of the health care provider to offer labor analgesia are main factors. Moreover, misconceptions like long-term backache, harm to baby, breastfeeding problem, increased cesarean section, slow labor progress, and permanent medical problems for the mother and newborn are some of the factors that affect utilization of labor analgesia. ${ }^{7,17,18}$

Obstetric analgesia services in the developing world are poor. Lack of the services results in mothers in unmeasured suffering, let alone analgesia. Almost all women would request analgesia during labor to be free of pain. In many health facilities situation there may not have analgesia for labor pain management. ${ }^{19}$ When analgesia is offered to women during labor and delivery, there would be greater satisfaction with their overall childbirth experience. From EDHS findings in 2014, of the $57.2 \%$ of Ethiopian pregnant women who attended at least one antenatal care visit, only $14.5 \%$ of women delivered in health facilities. In this context, pain relief during labor could be a critical incentive for increasing facility-based deliveries. ${ }^{20}$ There are few published data on the prevalence and determinants of labor pain management in Ethiopia. In addition, there is no study that documents the use of labor analgesia and its associated factors in the study area. So, this study was carried out with the aim to determine and document the practice of obstetric analgesia in labor pain management among obstetric caregivers in public health facilities of Kembata Tembaro zone (KTZ), Southern Ethiopia, for further study.

\section{Materials and Methods}

A facility-based cross-sectional study design was employed from March to April 2017 at public health facilities of KTZ, Southern Ethiopia. The Zone has seven woredas (districts) and one city administration and is located $228 \mathrm{~km}$ from Addis Ababa. According to 2007 census conducted by CSA, the Zone has a total population of 1,080,837 of whom about 536,676 are males and 544,161 are females with an area of 
$1,355.89 \mathrm{~km}^{2}$. The zone has a population density of 502.13 while 97,797 or $14.36 \%$ are urban inhabitants. ${ }^{21}$

Currently, in the KTZ, there are 4 hospitals (from which one is general hospital and three are primary hospitals), 35 health centers, and 1 gynecologist, 435 heath officers, 19 general practitioners, 438 nurses, and 134 midwives, and 7 integrated emergency surgery and obstetrics working in the institutions. According to the zone's report, there were 23,179 institutional deliveries in 2016 . The health facilities have 366 professionals giving maternal and newborn care services.

The study participants were health care providers who utilized obstetric analgesia and gave maternal care service in labor and delivery in public health facilities of KTZ. Since there were small numbers of source population in the study area, obstetric caregivers available during the data collection period were included conveniently in the study.

\section{Measurements}

Data were collected using a structured, pretested, and selfadministered questionnaire prepared by adapting from different studies, which was developed in English language to be understood by every respondent. ${ }^{20,22}$ The questionnaire had four essential components related to obstetric analgesia utilization in labor pain management including providers' sociodemographic characteristics, perception-related questions, practice-related questions, and facility-related questions. One day orientation was given for five selected diploma clinical nurses who collected data.

Before the actual data collection, the pretest was done on 18 professionals at Shone hospital and appropriate corrections were made before using it for the main study. The questionnaire was intended to collect information on sociodemographic characteristic of health care providers, perception-related questions, practice-related questions, and facility-related questions. The care providers were requested to complete the questionnaire following informed consent.

\section{Data analysis}

Data consistency was checked and entered into Epidata version 3.1 and then was exported to SPSS version 20 for further analysis and was summarized by using descriptive statistics. Tables and figures were used for data presentation. Bivariate logistic regression was used to identify factors associated with utilization of obstetric analgesia among obstetric caregivers based on adjusted odds ratio (AOR), 95\% CI, and $P$-value $<0.25$.
We used the enter approach in for inclusion into the multivariate model, while the Hosmer-Lemeshow statistic was used for model diagnostics. Multivariate logistic regression model was used to control the possible effect of confounders, and finally the variables that had independent association with utilization of obstetric analgesia were identified on the basis of AOR, with $95 \% \mathrm{CI}$ and $P$-value $<0.05$. The variables were entered to the multivariate model using the backward logistic regression.

\section{Ethical considerations}

Ethical clearance was obtained from ethical review board of the Institute of Health Sciences, Jimma University, to conduct the study. Permission to conduct the study in each health facility was secured from the respective health institutions in KTZ. Written informed consent was obtained from respondents after giving them information about the study. In addition, all the responses were kept confidential and anonymous.

\section{Results}

Out of the 366 questionnaires administered to health care providers, 340 of them returned the questionnaires making a response rate of $93 \%$. Table 1 shows the sociodemographic characteristics of the participants. About 220 (64.7\%) were females; 164 professionals $(48.2 \%)$ were protestant in religion. Out of the total respondents, $143(42.1 \%)$ were health officers, 137 (40.3\%) of respondents had experience between 6 and 10 years, and $222(65.3 \%)$ of them were BSc in qualification.

\section{Utilization of obstetric analgesia}

The finding shows that a total of $129(37.9 \%)$ respondents used any form of labor analgesia to clients in labor as shown in Figure 1.

Psychological support was the most commonly used/ practiced nonpharmacological labor pain management by $109(85.4 \%)$ health care providers, while $22(17.1 \%)$ prescribed Tramadol from systemic opioids analgesic, 14 $(10.8 \%)$ respondents used Diclofenac from nonopioids systemic analgesics. The overall utilization of the nonpharmacological and pharmacological methods by the health care providers were $186(54.7 \%)$ and $154(45.2 \%)$, respectively (Table 2 ).

Regarding the pattern/frequency of labor analgesia usage, only one health care provider used it routinely, $72(21.1 \%)$ of the respondents used it sometimes, and $56(16.5 \%)$ of them used it on maternal request. Two hundred eleven (62.1\%) 
Table I Sociodemographic characteristics of participants in public facilities in Kembata Tembaro Zone, Southern Ethiopia, March-April 2017 ( $\mathrm{N}=340)$

\begin{tabular}{|l|l|l|l|}
\hline \multicolumn{2}{|l|}{ Characteristics } & Frequency & $\%$ \\
\hline Sex & Male & 120 & 35.3 \\
& Female & 220 & 64.7 \\
& Protestant & 164 & 48.2 \\
& Orthodox & 112 & 32.9 \\
& Catholic & 21 & 6.2 \\
Profession & Muslim & 43 & 12.7 \\
& Medical doctor & 11 & 3.2 \\
& Health officer & 143 & 42.1 \\
Duration of & Nurse & 46 & 13.5 \\
practice/years & Midwife & 133 & 39.1 \\
of service & Integrated emergency & 7 & 2.1 \\
& surgery and obstetric & & \\
& $\leq 5$ & 104 & 30.6 \\
Qualification & 6-10 & 137 & 40.3 \\
& II-15 & 73 & 21.5 \\
& I6-20 & 19 & 5.6 \\
& Diploma & 7 & 2.0 \\
& BSc & 101 & 29.7 \\
& Masters & 222 & 65.3 \\
& General practitioner & 7 & 2.1 \\
& Gynecologist & 1 & 2.6 \\
& & & 0.3 \\
\hline
\end{tabular}

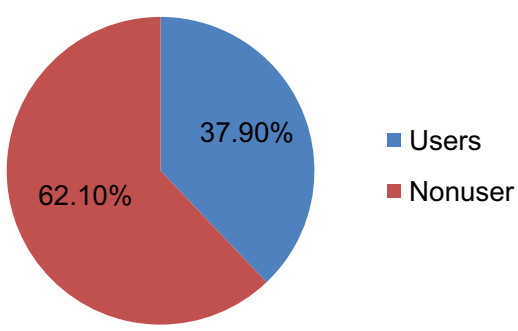

Figure I Percentage distribution of usage of any form of labor analgesia by respondents in public health facilities in Kembata Tembaro Zone, Southern Ethiopia, March-April 2017 ( $\mathrm{N}=340)$.

of care providers never used/practiced labor analgesia for laboring mothers (Figure 2).

\section{Knowledge of labor analgesia users/ provider in KTZ, Southern Ethiopia}

About 162 (47.6\%) participants had knowledge about labor analgesia. One hundred sixty (47.1\%) of them responded that pharmacological method is more effective than nonpharmacological method. About 158 (46.5\%) study subjects said that
Table 2 Types of labor analgesia offered by health care providers in Kembata Tembaro Zone, Southern Ethiopia, March-April 2017 $(n=129)$

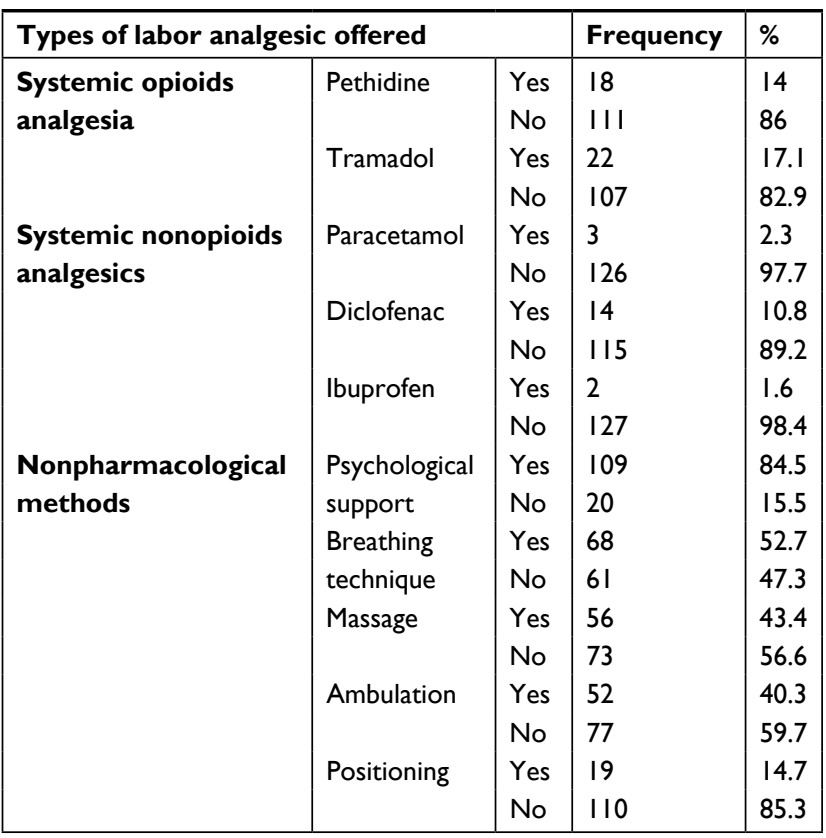

laboring mothers experience moderate to severe pain. Nearly half percent, 167 (49.1\%), of the participants responded that nonpharmacological method of pain relief can produce harmful effects on newborn. One hundred sixty-one (47.3\%) and $159(46.8 \%)$ of study subjects answered as continuous support of labor is associated with shorter of labor and labor is a natural process, respectively.

On the other hand, based on the composite knowledge score, about $162(47.6 \%)$ respondents had adequate knowledge about labor analgesia, while 178 (52.4\%) had inadequate knowledge as shown in Table 3.

\section{Attitude of labor analgesia users in KTZ, Southern Ethiopia}

Regarding the attitude of the respondents, 179 (52.6\%) of the study subjects agreed that using labor analgesia influences the progress of labor, while more than half percent, 180 (52.9\%), of the respondents believed that use of labor analgesia causes fetal distress. One hundred seventy-seven (52.1\%) of the study participants agreed that women should endure the natural labor pain. About 176 (51.8\%) of respondents admitted that using obstetric labor analgesia causes respiratory distress. The finding also revealed that about 147 (43.2\%) of the respondents agreed that women need pain relief during labor and childbirth. More 
Patterns of obstetric analgesia provision

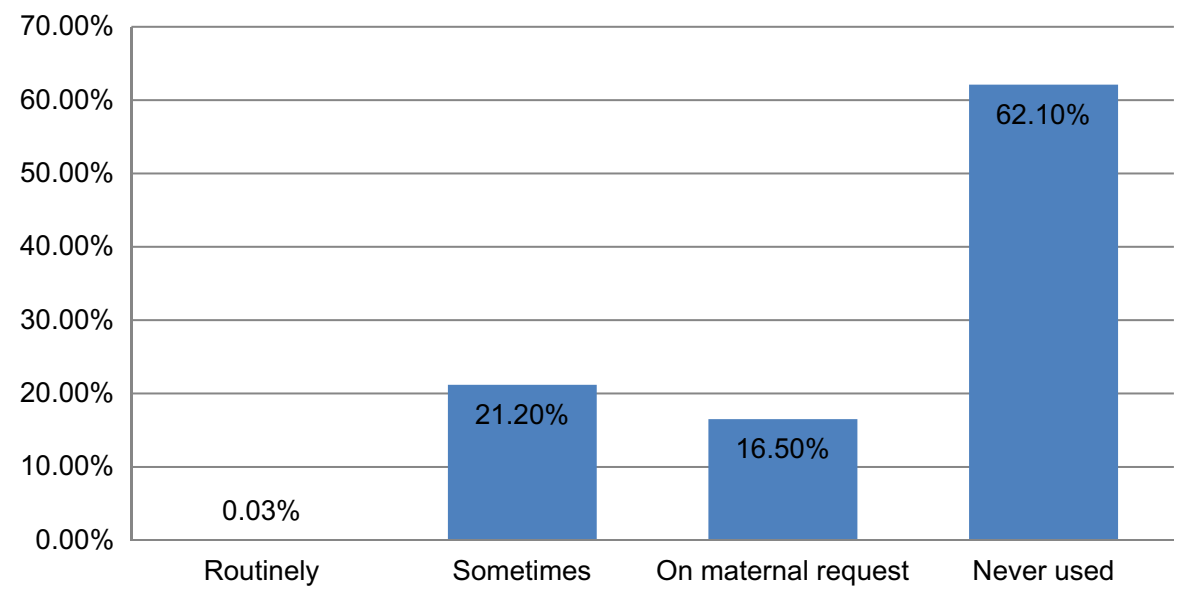

Figure 2 Patterns of analgesia provision by respondents' public health facilities in Kembata Tembaro Zone, Southern Ethiopia, March-April 20I7 ( $\mathrm{N}=340$ ).

Table 3 Knowledge of obstetric labor analgesia providers in public health facilities, Kembata Tembaro Zone, Southern Ethiopia, March-April 2017 ( $\mathrm{N}=340)$

\begin{tabular}{|l|l|l|l|}
\hline Knowledge question & Response & Frequency & $\%$ \\
\hline Do you know obstetric & I. Yes & 162 & 47.6 \\
labor analgesia? & 2. No & 178 & 52.4 \\
Is pharmacological & I. Yes & 160 & 47.1 \\
method of pain relief & 2. No & 168 & 49.4 \\
more effective than & 3. I do not know & 12 & 3.5 \\
nonpharmacological & & & \\
methods? & & 158 & \\
Are laboring clients & I. Yes & 163 & 46.5 \\
experiencing moderate & 2. No & 19 & 47.9 \\
to severe pain? & 3. I do not know & 167 & 5.6 \\
Can & I. Yes & 160 & 49.1 \\
nonpharmacological & 2. No & 13 & 47.1 \\
method of pain relief & 3. I do not know & & 3.8 \\
produce harmful & & 161 & \\
effects in the newborn? & & 163 & 47.3 \\
Is continuous support & I. Yes & 2. No & 48.2 \\
in labor associated & 3. I do not know & 16 & 2.6 \\
with shorter labors? & I. Yes & 2. No & 3. I do not know \\
Is laboring a natural & 9.6 \\
process? & & & \\
& & 159 & \\
\hline
\end{tabular}

than half of study participants, $179(52.6 \%)$, believed that use of labor analgesia causes late presentation. About 156 (45.9\%) of the study subjects agreed that labor analgesia offers better birth experience. One hundred fifty-nine (46.8\%) of the respondents believed that labor pain relief should include awareness creation and education for client and maternal care providers.

Overall, about $222(65.3 \%)$ of respondents had negative attitude, while $118(34.7 \%)$ of them had positive attitude toward obstetric labor analgesia (Table 4).

\section{Facility-related factors}

The main facility-related reason that hindered from using obstetric labor analgesia by respondents routinely was nonavailability of drugs (241 [70.9\%]). Similarly, all of the participants responded that lack of trained health care providers, guidelines and protocols were the most common bottlenecks for utilizing the methods to relief labor pain (Figure 3).

\section{Factors associated with utilization of labor analgesia among obstetric caregivers in KTZ, Southern Ethiopia, March-April 2017}

In the bivariate logistic regression analysis, utilization of labor analgesia was significantly associated with, qualification of the respondents, their service year, knowledge, attitude of the study subjects and availability of analgesia drugs by using enter method to identify the independent predictors of labor analgesia utilization. (Table 5) showed the multivariate logistic regression analysis; utilization of labor analgesia was significantly associated with knowledge, attitude and availability of drugs.

The result of multivariable logistic regression analysis showed that professionals who had inadequate knowledge were almost four (3.93) times more likely to use obstetric analgesia than those who had adequate knowledge (AOR: 3.93, 95\% CI: 2.28, 6.83). Statistical association also depicted that professionals' attitude had a significant association with labor analgesia, where professionals who had positive attitude were 4.35 times more likely to use obstetric analgesia than who had negative attitude (AOR: 4.35, 95\% CI 2.47, 7.67). Availability of labor analgesia drugs is also significantly 
Table 4 Attitude of obstetric labor analgesia providers in public health facilities, Kembata Tembaro Zone, Southern Ethiopia, MarchApril $2017(\mathrm{~N}=340)$

\begin{tabular}{|c|c|c|c|c|c|c|}
\hline \multirow[t]{2}{*}{ Attitude assessment items } & \multicolumn{2}{|c|}{ Disagree } & \multicolumn{2}{|c|}{ Neutral } & \multicolumn{2}{|c|}{ Agree } \\
\hline & No. & $\%$ & No. & $\%$ & No. & $\%$ \\
\hline Use of analgesia can influence the progress of labor & 156 & 45.9 & 5 & 1.5 & 179 & 52.6 \\
\hline Use of labor analgesia can cause fetal distress & 155 & 45.6 & 5 & 1.5 & 180 & 52.9 \\
\hline Women should endure the natural labor pain & 158 & 46.5 & 7 & 2.1 & 175 & 51.4 \\
\hline Use of labor analgesia can cause respiratory distress & 157 & 46.2 & 10 & 2.9 & 173 & 50.9 \\
\hline Women need pain relief during labor and childbirth & 187 & 55.0 & 6 & 1.8 & 147 & 43.2 \\
\hline Use of labor analgesia causes late presentation & 153 & 45.0 & 8 & 2.4 & 179 & 52.6 \\
\hline Labor analgesia offers a better birth experience & 181 & 53.2 & 3 & 0.9 & 156 & 45.9 \\
\hline $\begin{array}{l}\text { Labor pain relief services should include awareness creation } \\
\text { and education for client and care provider }\end{array}$ & 177 & 52.0 & 4 & 1.2 & 159 & 46.8 \\
\hline
\end{tabular}

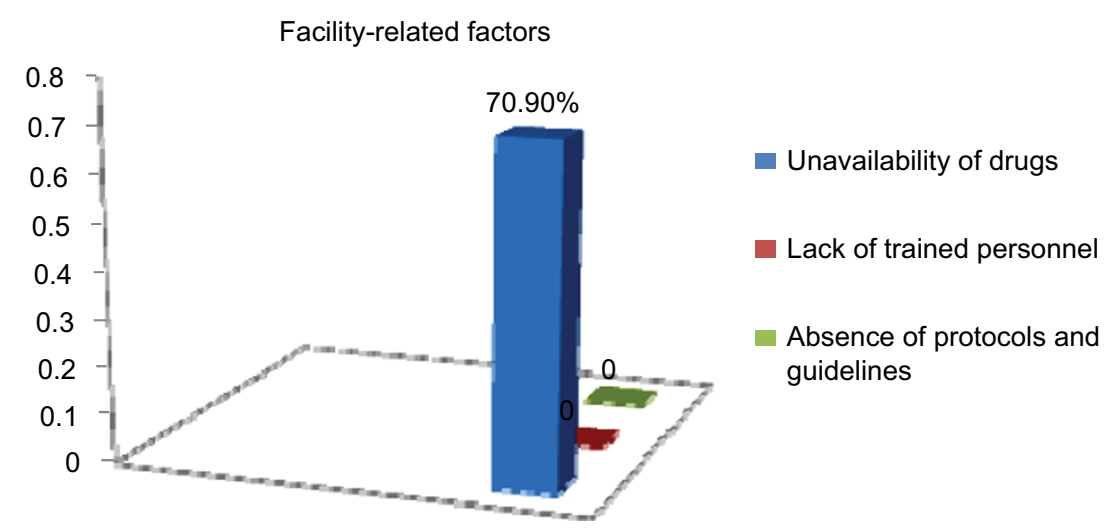

Figure 3 Facility-related factors that affect utilization of labor analgesia in public health facilities in Kembata Tembaro Zone, Southern Ethiopia, March-April 20I7 ( $\mathrm{N}=340$ ).

Table 5 Factors associated with utilization of labor analgesia among obstetric caregivers in Kembata Tembaro Zone, Southern Ethiopia, March-April 2017

\begin{tabular}{|c|c|c|c|c|c|}
\hline \multicolumn{2}{|l|}{ Variables } & \multicolumn{2}{|c|}{$\begin{array}{l}\text { Utilization of } \\
\text { labor analgesia }\end{array}$} & \multirow[t]{2}{*}{ COR } & \multirow[t]{2}{*}{ AOR } \\
\hline & & Yes & No & & \\
\hline \multirow[t]{3}{*}{ Qualification } & Lower level & 7 & 75 & 1 & 1 \\
\hline & Medium level & 116 & 134 & $9.3(4.1,20.92)^{a}$ & $0.07(0.01,1.56)$ \\
\hline & Higher level & 6 & 2 & $32.1(5.43,19.21)^{\mathrm{a}}$ & $0.40(0.61,2.69)$ \\
\hline \multirow[t]{2}{*}{ Attitude } & Negative & $14 \mid$ & 70 & $\mathrm{I}$ & 1 \\
\hline & Positive & 44 & 85 & $3.89(2.45,6.18)^{\mathrm{a}}$ & $4.35(2.47,7.67)^{b}$ \\
\hline \multirow[t]{5}{*}{ Years of service } & $\leq 5$ years & 43 & 61 & $0.28(0.052,1.52)$ & $0.23(0.04,1.42)$ \\
\hline & $6-10$ years & 51 & 86 & $0.24(0.04,1.26)$ & $0.24(0.04,7.43)$ \\
\hline & $11-15$ years & 22 & 51 & $0.17(0.031,0.95)^{\mathrm{a}}$ & $0.21(0.034,1.349)$ \\
\hline & 16-20 years & 8 & 11 & $0.29 \mid(0.045, I .89)$ & $0.35(0.05,2.70)$ \\
\hline & $\geq 21$ years & 5 & 2 & $\mathrm{I}$ & 1 \\
\hline \multirow[t]{2}{*}{ Knowledge } & Inadequate & 138 & 73 & $4.21(2.63,6.72)^{\mathrm{a}}$ & $3.94(2.28,6.83)^{\mathrm{b}}$ \\
\hline & Adequate & 40 & 89 & 1 & 1 \\
\hline \multirow{2}{*}{$\begin{array}{l}\text { Availability of drugs and } \\
\text { equipment }\end{array}$} & Not available & 67 & 168 & $0.27(0.17,0.45)^{\mathrm{a}}$ & $0.35(0.2,0.63)^{b}$ \\
\hline & Available & 62 & 43 & 1 & 1 \\
\hline
\end{tabular}

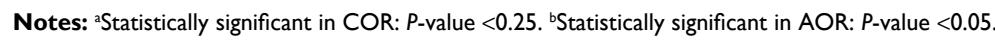

Abbreviations: AOR, adjusted odds ratio; COR, crude odds ratio. 
associated with utilization of obstetric analgesia, when drugs were not readily accessible in the facility, health care providers were $65 \%$ times less likely to use labour analgesia, than when drugs were freely available for use (AOR: 0.35, 95\% CI: $0.2,0.63)$.

\section{Discussion}

This study revealed that a substantial majority (211 [62.1\%]) of health care providers in public health facilities of KTZ do not use obstetric analgesia to manage labor pain. Indeed, to some, it was an entirely new concept, while only one of respondent administered labor analgesia routinely; $72(21.2 \%)$ of the study participants practiced it sometimes and $56(16.5 \%)$ of the respondents offered labor analgesia on maternal request. Overall, 37.9\% of respondents had provided any form of labor analgesia to manage labor pain.

These findings are not encouraging, especially when it is expected that women should be offered, among other things, effective pain relief during labor. However, it is consistent with the finding in Benin (38.9\%). ${ }^{22}$ On the other hand, this finding is lower than the studies conducted in Nigeria, ${ }^{7,23}$ which were $48.4 \%$ and $49 \%$, respectively. The discrepancy might be due to well-organized health facilities, the existence of better education, cultural, and socioeconomic status of Nigeria, which might be the reason to use the labor analgesia to relieve labor pain.

The findings of this study showed the proportion of both nonpharmacological (54.7\%) and pharmacological (45.2\%) methods of pain relief in labor. The finding is comparable with the nonpharmacological analgesia methods in Nigeria $(56.8 \%) .{ }^{22}$ But, this study result is higher than the studies performed in Bangladesh and Amhara region referral hospitals which were $40 \%$ and $40.1 \%$, respectively. ${ }^{3,20}$ This difference might be due to the setting of the study area, which included all health facilities and the sample size. In addition, in referral hospitals, there might be high client flow, lack of time, and intermittent appearance and disappearance of health care providers including obstetricians, midwives, nurses, and students of various professions like residents, interns, health officers, nurses, and midwives, who might be there to learn. Meanwhile, they may not give attention to labor pain and may not use labor analgesia to manage labor pain., 3

The result of this study is comparable with the findings of the pharmacological methods in Ethiopia (45\%) ${ }^{17}$ but higher than the studies performed in Addis Ababa (35.6\%). ${ }^{25}$ This difference might be due to current study sample size and the study settings. The other justification might be the consideration of opioids as safe, noninvasive, easy to administer, and not requiring monitoring or the presence of an anesthetist. ${ }^{17}$ Tramadol injection was offered by most of the respondents among the systemic forms of pharmacological management of labor pain (32 [24.6\%]).

This finding is consistent with the study done in India, as Tramadol does not induce respiratory distress in the newborn unlike other opioids. ${ }^{26}$ But in other studies performed in different parts of the world, pethidine injection is offered by most of the respondents. ${ }^{7,25,27,28}$ The respondents might not have used or practiced pethidine in this study, may be due to pethidine causing more side effect than other opioids. ${ }^{29}$ This study also assessed systemic nonopioids analgesics (paracetamol, Diclofenac, and ibuprofen) offered by $17.7 \%$ of respondents. This finding is comparable with the study done in Addis Ababa. ${ }^{25}$

According to the respondents, the reason for not using pain relief routinely might be the negative attitude of professionals about labor pain management, inadequate knowledge, lack of skilled professionals, unavailability of analgesic drugs, and lack of protocol to use labor analgesia during labor and delivery. It is consistent with the studies done in Nigeria, Amhara region and Lesotho. , $^{720,30}$

According to the analytic part of this study, inadequate knowledge, positive attitude, and unavailability of analgesia drugs were statistically significant predictors of utilization of labor analgesia. Moreover, the result of the study showed that professionals who had inadequate knowledge were 3.9 times more likely to use obstetric analgesia than who had adequate knowledge (AOR: 3.9, 95\% CI: 2.27, 6.83). The study done in Amhara region depicted that those obstetric caregivers who had inadequate knowledge were three times (2.57) more likely to use obstetric analgesia than professionals who had adequate knowledge (AOR: 2.57, 95\% CI: 1.42, 4.65).$^{20}$ The study conducted in Nigeria and Uganda supported this result in which in low- and middle-income countries majority of the obstetric caregivers were not taught well about labor analgesia during their training program, and their practical exposure to it was very limited., ${ }^{714}$

Since this study is concerned about pharmacological (opioids) and nonpharmacological methods, adequate knowledge of professionals was found to be protective for obstetric analgesia use in labor pain management. Nevertheless, most professionals who had lower qualification (diploma) and some of the medium professionals (BSc) may not know the methods that are known to some medium and higher professionals. Besides this, the mostly utilized method of labor 
analgesia was nonpharmacological than pharmacological. So this implication is reflected by their utilization of what they know (nonpharmacological).

Professionals' attitude is significantly and positively associated with labor analgesia, where professionals who had positive attitude were 4.21 times more likely to use obstetric analgesia than who had negative attitude (AOR: 4.21, 95\% CI 2.42, 7.32). In this study, negative attitude was one of the factors for not using obstetric analgesia by the respondents. The commonest reasons adduced were fear of respiratory distress, delay in the progress of labor, fear of causing fetal distress, and that women can endure natural pain of labor. The finding was consistent with the study conducted in Bangladesh, Nigeria, and Ethiopia. ${ }^{3,7,17}$

The study result also showed that availability of labor analgesia drugs is also significantly associated with utilization of obstetric analgesia, when there is unavailability of drugs in the facility; so there could be $65 \%$ less likelihood of usage of labor analgesia among health care providers (AOR: $0.354,95 \% \mathrm{CI}: 0.2,0.63)$. This result is consistent with the study finding of Addis Ababa.

Absence of guidelines and protocols, shortage of skilled personnel, and shortage of drugs were among the factors mentioned by participants for not practicing/using obstetric analgesia.

Qualification of the respondents was not significantly associated in this study, but it was significant in other studies. Also, years of service of the respondents was not significantly associated in this study and other studies as well. ${ }^{11,25}$

\section{Conclusion}

The study revealed that the proportion of obstetric analgesia utilization in labor pain management was low in KTZ public health facilities. Inadequate knowledge, positive attitude, and unavailability of analgesia drugs in the study facilities were significantly associated with utilization of labor analgesia. Unfortunately, health care givers in KTZ are not yet routinely providing pain relief to all laboring mothers during labor in a well-established way. Unavailability of drugs, lack of skilled personnel to practice standard analgesia technique, and lack of guidelines and protocols were barriers adduced by the respondents. Besides this, inadequate knowledge and negative attitude toward obstetric labor analgesia were the reasons for not using analgesia to manage labor pain. The poor practice of labor analgesia in the study area will notify all obstetric caregivers and other responsible bodies of health institutions to introduce pain relief services in a systematic way into the health facilities.

\section{Recommendations}

- Southern Nations, Nationalities and People's Regional State Health Bureau, and KTZ Health Department are responsible for developing national protocol and guidelines on obstetric analgesia for obstetric caregivers that should be available at any level.

- Provision of training and supply of required drugs will help care providers to fulfill the gap between provision of analgesics in labor pain and their knowledge, attitude, and skill that can be achieved through teamwork by all the stakeholders in the health sector.

- Further studies should consider gathering more data from a more diverse sample to address the generalized issue in different parts of the country and come up with more representative findings.

\section{Acknowledgments}

We would like to forward our gratitude to Jimma University, Institute of Health Sciences, School of Nursing and Midwifery. Our special thanks go to the supervisors, data collectors, and the respondents, and the Zonal Health Department. The research was funded by Jimma University.

\section{Author contributions}

TEG initiated the research project, wrote the research proposal, conducted the research, did data entry and analysis, and wrote the manuscript. AT and AGK were involved in the write-up of the proposal and manuscript. All authors contributed to data analysis, drafting or revising the article, gave final approval of the version to be published, and agree to be accountable for all aspects of the work.

\section{Disclosure}

The authors report no conflicts of interest in this work.

\section{References}

1. Gupta S, Anand Kumar GS, Singhal H. Acute pain - labour analgesia. Indian J Anaesth. 2006;50(5):363-369.

2. Othman M. Inhaled analgesia for labor pain. IOSR J Pharm. 2016;6(6):16-26.

3. Tasnim S. Perception about pain relief during normal labour among health care providers conducting delivery. Med Today. 2010;22(1):20-23.

4. Ebirim LN, Buowari OY, Ghosh S. Physical and psychological aspects of pain in obstetrics. In: Ghosh S, editor. Pain in Perspective. London, UK: InTech; 2012.

5. Jones L, Othman M, Dowswell T, et al. Pain management for women in labour: an overview of systematic reviews. Cochrane Database Syst Rev. 2012;7(3):CD009234.

6. Karn S, Yu H, Karna S, Chen L, Qiao D. Women's awareness and attitudes towards labor analgesia influencing practice between developed and developing countries. Adv Reprod Sci. 2016;4(2):46-52. 
7. Ogboli-Nwasor E, Adaji S, Bature S, Shittu O. Pain relief in labor: a survey of awareness, attitude, and practice of health care providers in Zaria, Nigeria. J Pain Res. 2011;4:227-232.

8. Kuti O, Faponle AF. Perception of labour pain among the Yoruba ethnic group in Nigeria. J Obstet Gynaecol. 2006;26(4):332-334.

9. Food, Medicine and Healthcare Administration and Control Authority of Ethiopia. Standard Treatment Guidelines For General Hospital: Good Prescribing \& Dispensing Practices for Better Health Outcomes. 2014. Available from: http://apps.who.int/medicinedocs/documents/s21694en/ s21694en.pdf. Accessed March, 2016.

10. Hodnett ED, Gates S, Hofmeyr GJ, Sakala C. Continuous support for women during childbirth. Cochrane Database Syst Rev. 2013;7:CD003766.pub5

11. Apondi C. A Survey of the Knowledge Attitude and Practice Regarding Epidural Labor Analgesia among Obstetricians at the Kenyatta National Hospital [master's thesis]. Kenya: University of Nairobi; 2012.

12. Ding T, Wang DX, Qu Y, Chen Q, Zhu SN. Epidural labor analgesia is associated with a decreased risk of postpartum depression: a prospective cohort study. Anesth Analg. 2014;119(2):383-392.

13. Cochrane AR. Perceptions of Labor and Delivery Clinicians on Nonpharmacological Methods for Pain Relief During Labor; 2015. Available from: https://digitalcommons.brockport.edu/cgi/viewcontent.cgi?article $=1093 \&$ context $=$ honors. Accessed March, 2016 .

14. Nabukenya MT, Kintu A, Wabule A, Muyingo MT, Kwizera A. Knowledge, attitudes and use of labour analgesia among women at a low-income country antenatal clinic. BMC Anesthesiol. 2015;15:98.

15. Kaur S, Charanjit S, Yahya N. Combined spinal-epidural analgesia in labour: its effects on delivery outcome. Braz J Anesthesiol. 2016;66(3):259-264.

16. [No authors listed]. ACOG Committee Opinion \#295: pain relief during labor. Obstet Gynecol. 2004;104(1):213.

17. McCauley M, Stewart C, Kebede B. A survey of healthcare providers' knowledge and attitudes regarding pain relief in labor for women in Ethiopia. BMC Pregnancy Childbirth. 2017;17(1):56.

18. Reena, Bandyopadhyay KH, Afzal M, Mishra AK, Paul A. Labor epidural analgesia: Past, present and future. Indian J Pain. 2014;28(2):71-81.
19. Size M, Soyannwo OA, Justins DM. Pain management in developing countries. Anaesthesia. 2007;62(Suppl 1):38-43.

20. Bitew A, Workie A, Seyum T, Demeke T. Utilization of obstetric analgesia in labor pain management and associated factors among obstetric care givers in Amhara Regional State Referral Hospitals, Northwest Ethiopia: a hospital-based cross-sectional study. J Biomed Sci. 2016;5:2.

21. Ethiopia Office of the Population and Housing Census Commission. Summary and Statistical Report of the 2007 Population and Housing Census: Population Size by Age and Sex. Addis Ababa: Federal Democratic Republic of Ethiopia, Population Census Commission; 2008.

22. Imarengiaye $\mathrm{CO}$. Trends in pain relief in labour: implications for obstetric analgesia service in Nigeria. Niger Postgrad Med J. 2005;12(3):193-202.

23. Lawani LO, Eze JN, Anozie OB, Iyoke CA, Ekem NN. Obstetric analgesia for vaginal birth in contemporary obstetrics: a survey of the practice of obstetricians in Nigeria. BMC Pregnancy Childbirth. 2014;14:140.

24. Goodson C, Martis R. Pethidine: to prescribe or not to prescribe? A discussion surrounding pethidine's place in midwifery practice and New Zealand prescribing legislation. NZCOM J. 2014;49:23-28.

25. Mulugeta $\mathrm{H}$. Practice of labor analgesia and its perceived barriers among health providers working in public hospitals of Addis Ababa, Ethiopia; 2016; Addis Ababa.

26. Bhuvaneshwari K, Rengasamy CK. Attitude of obstetricians regarding labour analgesia and limitations in practising it. Int J Reprod Contracept Obstet Gynecol. 2017;6(2):388-391.

27. Bricker L, Lavender T. Parenteral opioids for labor pain relief: a systematic review. Am J Obstet Gynecol. 2002;186(5 Suppl Nature):S94-S109.

28. Furmanik J. Labour epidural analgesia in Poland in 2009 - a survey. Anaesthesiol Intensive Ther. 2013;45(3):149-152.

29. Almushait M, Ghani RA. Perception toward non-pharmacological strategies in relieving labor pain: an analytical descriptive study. $J$ Nat Sci Res. 2014;4(2):5-12.

30. Roets L, Moru MM, Nel M. Lesotho midwives' utilization of nonpharmacological pain management methods during the first of stage labour. Curationis. 2005;28(3):73-77.
Journal of Pain Research

\section{Publish your work in this journal}

The Journal of Pain Research is an international, peer reviewed, open access, online journal that welcomes laboratory and clinical findings in the fields of pain research and the prevention and management of pain. Original research, reviews, symposium reports, hypothesis formation and commentaries are all considered for publication

\section{Dovepress}

The manuscript management system is completely online and includes a very quick and fair peer-review system, which is all easy to use. Visit http://www.dovepress.com/testimonials.php to read real quotes from published authors. 